\title{
Determinants of Laplacians *
}

\author{
Peter Sarnak \\ Mathematics Department, Stanford University, Stanford, CA 94305, USA
}

\begin{abstract}
The determinant of the Laplacian on spinor fields on a Riemann surface is evaluated in terms of the value of the Selberg zeta function at the middle of the critical strip. A key role in deriving this relation is played by the Barnes double gamma function.
\end{abstract}

1.

In recent years the problem of evaluating the determinants of certain elliptic operators has received considerable attention. The case of determinants of Laplacians on Riemann surfaces is crucial in Polyakov's string theory [P]. It also comes up in Ray-Singer's analytic torsion [R-S]. In recent papers D'Hoker and Phong [D-P 1,2] and Kierlanczyk [KIE] computed these determinants of Laplacians in terms of special values of the Selberg zeta function. They succeeded except in the case of the Laplacian corresponding to the Dirac operator on spinors, for which they conjectured the determinant should be related to the Selberg zeta function at the middle of the critical strip. Our aim in this note is to prove that this is indeed so. The method followed by the above authors is to use the precise heat kernel for the hyperbolic plane, and the Selberg zeta function appears somewhat surprisingly. Our approach is more in the spirit of the analysis of the Selberg zeta function given in Hejhal [H], Selberg [S], and Vignéras [VI]. We show that the Selberg zeta function may be realized as a functional determinant (in fact as an analogue of a characteristic polynomial). The precise statement is given in Theorem 1. Our result follows from an asymptotic analysis in which a key role is played by Barnes' double gamma function and in particular Stirlings formula. The determinants in question are computed by specializing the parameter in Theorem 1.

Our notation will be as in D'Hoker and Phong [D-P 2]. We review it briefly. $M$ is a compact Riemann surface of genus $h \geqq 2$ carrying a metric $d s^{2}$ of constant

^ This work was supported in part by the NSF Grant No. DMS-85-04329 
curvature -1 . Let $z$ be a local conformal variable and $d s^{2}=\varrho|d z|^{2}$. Let $T^{n}$ be the space of tensors $f(z)(d z)^{n}$ on $M$. We also allow $n$ to be half an integer by choosing a spinor structure. Thus $T^{0}$ denotes the space of functions and $T^{1 / 2}$ a space of spinors. Define operators $\nabla_{n}: T^{n} \rightarrow T^{n-1}$ and $\nabla^{n}: T^{n} \rightarrow T^{n+1}$ by

$$
\nabla_{n}=\varrho^{-1} \bar{\partial}, \quad \nabla^{n}=\varrho^{n} \partial \varrho^{-n},
$$

then

$$
\nabla_{n}^{*}=-\nabla^{n-1}, \quad\left(\nabla^{n}\right)^{*}=-\nabla_{n+1} .
$$

The Laplacians $\Delta_{n}^{+}$and $\Delta_{n}^{-}$on $T^{n}$ are defined by

$$
\Delta_{n}^{+}=-\nabla_{n+1} \nabla^{n}, \quad \Delta_{n}^{-}=-\nabla^{n-1} \nabla_{n} .
$$

Thus $\Delta_{0}=\Delta_{0}^{ \pm}$is the Laplacian on functions and $\Delta_{1 / 2}^{-}$is the Laplacian corresponding to the Dirac operator $\nabla_{1 / 2}$. The operators $\Delta_{n}^{ \pm}$are non-negative selfadjoint. If $B$ is such an operator and $0 \leqq \lambda_{0} \leqq \lambda_{1} \leqq \lambda_{2} \ldots$ its eigenvalues we define

$$
\operatorname{det} B=\prod_{j=0}^{\infty} \lambda_{j} .
$$

This product is regularized by the usual zeta function method (see Sect. 2). We also define

$$
\operatorname{det}^{\prime} B=\prod_{\lambda_{j} \neq 0} \lambda_{j}
$$

In order to make computations we make use of the uniformization theorem. $M$ may be realized as $\mathfrak{h} / \Gamma$, where $\mathfrak{h}$ is the upper half plane and $\Gamma$ a discrete subgroup of $\operatorname{PSL}(2, \mathbb{R})$. To give an explicit spinor structure on $M$, let $\tilde{\Gamma}$ be the subgroup of $S L(2, \mathbb{R})$ which projects to $\Gamma$. Let $\chi: \widetilde{\Gamma} \rightarrow\{ \pm 1\}$ be a character for which $\chi(-I)=-1$. We denote by $S(2 n)$ the space of automorphic forms $f$ on $\mathfrak{h}$ satisfying

$$
f(\tilde{\gamma} z)=(\chi(\tilde{\gamma}))^{2 n}\left(\frac{c z+d}{|c z+d|}\right)^{2 n} f(z), \quad \tilde{\gamma} \in \tilde{\Gamma},
$$

where $\tilde{\gamma}=\left(\begin{array}{ll}* & * \\ c & d\end{array}\right)$. We remark that our space $S(2 n)$ corresponds to the space $S(n)$ in D'Hoker and Phong [D-P 2]. We use this convention so as to be consistent with the notation in automorphic forms, e.g. [H]. With this convention $S(1)$ corresponds to the space of spinors. $S(2 n)$ is a Hilbert space with the inner product

$$
\langle f, g\rangle=\int_{\mathfrak{h} / \Gamma} f(z) \overline{g(z)} \frac{d x d y}{y^{2}} .
$$

$S(0)$ is, of course, the space of functions on $M$. The operators

$$
D_{2 n}=-y^{2}\left(\frac{\partial^{2}}{\partial x^{2}}+\frac{\partial^{2}}{\partial y^{2}}\right)+2 i n y \frac{\partial}{\partial x}
$$

are self-adjoint on $S(2 n)$. Furthermore, the isometry $I: T^{n} \rightarrow S(2 n)$ defined by

$$
f(z) \stackrel{I}{\leadsto} y^{n} f(z),
$$


conjugates

$$
\Delta_{n}^{+} \text {with } D_{2 n}+n(n+1) \text { and } \Delta_{n}^{-} \text {with } D_{2 n}+n(n-1) \text {, }
$$

see [D-P 2, pp. 538]. It follows that

$$
\begin{aligned}
\operatorname{det}^{\prime}\left(\Delta_{0}\right) & =\operatorname{det}^{\prime}\left(D_{0}\right), \\
\operatorname{det}^{\prime}\left(\Delta_{1 / 2}^{-}\right) & =\operatorname{det}^{\prime}\left(D_{1}-1 / 4\right) .
\end{aligned}
$$

Corresponding to forms of weight 0 and 1 we form the Selberg zeta functions

$$
Z_{v}(s)=\prod_{\gamma \text { primitive }} \prod_{k=0}^{\infty}\left(1-(\chi(\gamma))^{v} e^{-(k+s) l_{\gamma}}\right),
$$

$v=0,1$ and where $\gamma$ runs over the primitive conjugacy classes of $\tilde{\Gamma}$ (these correspond to the primitive closed geodesics on $M$ ). $l_{\gamma}$ is the length of the corresponding geodesic.

The Barnes double gamma function $\Gamma_{2}(s)$ is defined by the canonical product

$$
\frac{1}{\Gamma_{2}(s+1)}=(2 \pi)^{s / 2} e^{-s / 2-\frac{\gamma+1}{2} s^{2}} \prod_{k=1}^{\infty}\left(1+\frac{s}{k}\right)^{k} e^{-s+s^{2} / 2 k},
$$

$\gamma$ is Euler's constant. One checks that $\Gamma_{2}$ satisfies

$$
\Gamma_{2}(1)=1, \quad \Gamma_{2}(s+1)=\frac{\Gamma_{2}(s)}{\Gamma(s)}, \quad \Gamma_{2}(n+1)=\frac{1^{2} \cdot 2^{2} \ldots n^{n}}{(n !)^{n}} .
$$

Theorem 1.

$$
\begin{gathered}
\operatorname{det}\left(D_{0}+s(s-1)\right)=Z_{0}(s)\left(e^{E-s(s-1)} \frac{\left(\Gamma_{2}(s)\right)^{2}}{\Gamma(s)}(2 \pi)^{s}\right)^{2 h-2}, \\
\operatorname{det}\left(D_{1}+s(s-1)\right)=Z_{1}(s)\left(e^{E-s(s-1)}\left(\Gamma_{2}(s+1 / 2)\right)^{2}(2 \pi)^{s}\right)^{2 h-2},
\end{gathered}
$$

where $E=-\frac{1}{4}-\frac{1}{2} \log 2 \pi+2 \zeta^{\prime}(-1)$.

In the above theorem $\zeta(s)$ is the Riemann zeta function. The constant $\zeta^{\prime}(-1)$ comes through the relation $\zeta^{\prime}(-1)=\frac{1}{12}-\log A$, where

$$
\log A=\lim _{n \rightarrow \infty}\left(\sum_{k=1}^{n} k \log k-\left(\frac{n^{2}}{2}+\frac{n}{2}+\frac{1}{12}\right) \log n+\frac{n^{2}}{4}\right),
$$

see Vardi [VA 1]. $A$ is known as Kinkelin's constant [KIN]. ${ }^{1}$ This constant also appears in $\operatorname{det}^{\prime}(\Delta)$ where $\Delta$ is the Laplacian on $S^{2}$, see Vardi [VA 2].

\section{Corollary 1.}

$$
\begin{gathered}
\operatorname{det}^{\prime}\left(\Delta_{0}\right)=Z_{0}^{\prime}(1) e^{(2 h-2)\left(2 \zeta^{\prime}(-1)-1 / 4+1 / 2 \log 2 \pi\right)}, \\
\operatorname{det}^{\prime}\left(\Delta_{1 / 2}^{-}\right)=\frac{1}{(2 N) !} Z_{1}^{(2 N)}(1 / 2) e^{(4 g-4) \zeta^{\prime}(-1)},
\end{gathered}
$$

where $N$ is the dimension of the space of zero modes of the Dirac operator.

${ }^{1} A=1 \cdot 28242713 \ldots$ 
The value above for $\operatorname{det}^{\prime}\left(\Delta_{0}\right)$ agrees with D'Hoker and Phong [D-P 2]. The determinants $\operatorname{det}^{\prime}\left(\Delta_{n}^{ \pm}\right)$for all $n$ may be calculated from (1.10) and Theorem 1 by using the fact that the spectrum of $D_{2 n-2 l}, l \in \mathbb{Z}$ is essentially the same as that of $D_{2 n}$, as is easily seen via the operators $\nabla^{n}$ and $\nabla_{n}$ (the difference is merely a finite number of explicit eigenvalues coming from holomorphic forms of various weights, see Hejhal [H, p. 408, Theorem 5.6]).

2.

\subsection{Regularization of Determinants}

Let $\lambda_{0} \leqq \lambda_{1} \leqq \lambda_{2} \ldots$ be the eigenvalues of one of our Laplacians $B$. As is well known from differential equations, see Gilkey $[\mathrm{G}]$ for example,

$$
\widetilde{\theta}(t)=\sum_{n=0}^{\infty} e^{-\left(\lambda_{n}-\lambda_{0}\right) t}=\operatorname{TR}\left(e^{-\left(B-\lambda_{0} I\right) t}\right)
$$

satisfies

$$
\widetilde{\theta}(t) \sim \frac{\tilde{\alpha}}{t}+\widetilde{\beta}+\tilde{\gamma} t \ldots \quad \text { as } \quad t \rightarrow 0
$$

We begin by defining $\operatorname{det}(B+s(s-1))$ for $s$ real $s(s-1)>\lambda_{0}$. Consider

Formally,

$$
\begin{aligned}
H(w, s) & =\sum_{k=0}^{\infty}\left(\lambda_{k}+s(s-1)\right)^{-w} \text { for } \operatorname{Re}(w)>1 \\
& =\frac{1}{\Gamma(w)} \int_{0}^{\infty} \widetilde{\theta}(t) e^{-t\left(s(s-1)+\lambda_{0}\right)} t^{w} \frac{d t}{t} .
\end{aligned}
$$

$$
\left.\frac{\partial H}{\partial w}\right|_{w=0}=-\sum_{k=0}^{\infty} \log \left(\lambda_{k}+s(s-1)\right)
$$

so one defines

$$
\operatorname{det}(B+s(s-1))=e^{-\frac{\partial H}{\partial w}(0, s)}
$$

To see that this makes sense one shows that $H(w, s)$ is meromorphic in $w$ and regular at $w=0$. Indeed, a little manipulation shows that

$$
\begin{aligned}
H(w, s)= & \frac{1}{\Gamma(w)} \int_{0}^{1}\left(\tilde{\theta}(t)-\frac{\alpha}{t}-\beta\right) e^{-t\left(s(s-1)+\lambda_{0}\right)} t^{w} \frac{d t}{t} \\
& +\frac{1}{\Gamma(w)} \int_{1}^{\infty} \tilde{\theta}(t) e^{-t\left(s(s-1)+\lambda_{0}\right)} t^{w} \frac{d t}{t} \\
& +\frac{\tilde{\alpha}}{\Gamma(w)\left(s(s-1)+\lambda_{0}\right)^{w-1}}\left[\Gamma(w-1)-\int_{s(s-1)+\lambda_{0}}^{\infty} e^{-y} y^{w-1} \frac{d y}{y}\right] \\
& +\frac{\tilde{\beta}}{\Gamma(w)\left(s(s-1)+\lambda_{0}\right)^{w}}\left[\Gamma(w)-\int_{s(s-1)+\lambda_{0}}^{\infty} e^{-y} y^{w} \frac{d y}{y}\right] .
\end{aligned}
$$


From this the regularity of $H(w, s)$ at $w=0$ as well as the smoothness of $\frac{\partial H}{\partial w}(0, s)$ in $s$ for $s(s-1)>-\lambda_{0}$, is evident. We always interpret any $\prod_{k=0}^{\infty} \mu_{n}$ by the above regularization. Using this notation we have

$$
\operatorname{det}(B+s(s-1))=\prod_{k=0}^{\infty}\left(\lambda_{k}+s(s-1)\right)=\prod_{k=0}^{R}\left(\lambda_{k}+s(s-1)\right) \prod_{k=R+1}^{\infty}\left(\lambda_{k}+s(s-1)\right)
$$

for any $R$. Since the second factor is defined now for $s(s-1)>-\lambda_{R+1}$, while the first for all $s$ we may define $\operatorname{det}(B+s(s-1))$ for all real $s$. It is smooth in $s$ and vanishes only at those $s$ for which $\lambda_{k}+s(s-1)=0$ for some $k$. One could define $\operatorname{det}(B+s(s-1))$ for complex $s$ as well and show that this function is entire. We do not do so since it will follow from our considerations anyhow.

Returning to (2.2) and differentiating w.r.t.s.,

$$
\begin{gathered}
\frac{1}{2 s-1} \frac{\partial H}{\partial s}=\sum_{k=0}^{\infty}(-w)\left(\lambda_{k}+s(s-1)\right)^{-w-1} \\
\frac{1}{2 s-1} \frac{\partial}{\partial s}\left(\frac{1}{2 s-1} \frac{\partial H}{\partial s}\right)=w(w+1) \sum_{k=0}^{\infty}\left(\lambda_{k}+s(s-1)\right)^{-w-2} .
\end{gathered}
$$

Hence if $F(s)=\frac{\partial H}{\partial w}(0, s)=-\log \operatorname{det}(B+s(s-1))$, then

$$
\frac{d}{d s}\left(\frac{1}{2 s-1} \frac{d F}{d s}\right)=\sum_{k=0}^{\infty}\left(\lambda_{k}+s(s-1)\right)^{-2}(2 s-1)
$$

We will need to know the asymptotic behavior of $\operatorname{det}(B+s(s-1))$ as $s \rightarrow \infty$. If $\theta(t)$ is defined by

$$
\theta(t)=\sum_{k=0}^{\infty} e^{-\lambda_{k} t}
$$

then

$$
\theta(t) \sim \frac{\alpha}{t}+\beta+\gamma t \ldots \quad \text { as } \quad t \rightarrow 0
$$

for suitable constants $\alpha, \beta, \gamma$. We may write

$$
H(w, s)=\frac{1}{\Gamma(w)} \int_{0}^{\infty} \theta(t) e^{-s(s-1) t} t^{w} \frac{d t}{t},
$$

and if we split this integral as we did in (2.5), we get

$$
\begin{aligned}
\frac{\partial H}{\partial w}(0, s)= & F(s)=\int_{0}^{1} f(t) e^{-t s(s-1)} d t-\alpha s(s-1) \\
& +\alpha s(s-1) \log s(s-1)-\beta \log s(s-1)-\alpha s(s-1) \\
& \times \int_{s(s-1)}^{\infty} e^{-y} \frac{d y}{y^{2}}-\beta \int_{s(s-1)}^{\infty} e^{-y} \frac{d y}{y} .
\end{aligned}
$$


Here $f(t)=\frac{1}{t}\left(\theta(t)-\frac{\alpha}{t}-\beta\right)$ which is bounded. The first and last two terms of (2.9) tend to 0 as $s \rightarrow \infty$, hence

$$
\begin{aligned}
-\log \operatorname{det}(B+s(s-1))= & \alpha s(s-1) \log s(s-1)-\alpha s(s-1) \\
& -\beta \log s(s-1)+o(1) \text { as } s \rightarrow \infty .
\end{aligned}
$$

\subsection{Selberg Zeta Function}

On applying the Selberg Trace Formula in the case of $S(0)$ and $S(1)$ to the function $h(r)=\frac{1}{r^{2}+(s-1 / 2)^{2}}-\frac{1}{r^{2}+\beta^{2}}$, see Hejhal [H, pp. 448 and 72], we obtain the partial fraction expansion for $Z_{v}(s)$ as defined in (1.12).

$$
\begin{aligned}
\frac{1}{2 s-1} \frac{Z_{0}^{\prime}}{Z_{0}}(s)= & \frac{1}{2 \beta} \frac{Z_{0}^{\prime}}{Z_{0}}\left(\frac{1}{2}+\beta\right)+\sum_{n=0}^{\infty}\left(\frac{1}{\lambda_{n}^{(0)}+s(s-1)}-\frac{1}{\lambda_{n}^{(0)}-1 / 4+\beta^{2}}\right) \\
& +(2 h-2) \sum_{k=0}^{\infty}\left(\frac{1}{\beta+1 / 2+k}-\frac{1}{s+k}\right), \\
\frac{1}{2 s-1} \frac{Z_{1}^{\prime}}{Z_{1}}(s)= & \frac{1}{2 \beta} \frac{Z_{1}^{\prime}\left(\frac{1}{2}+\beta\right)}{Z_{1}\left(\frac{1}{2}+\beta\right)}+\sum_{n=0}^{\infty}\left(\frac{1}{\lambda_{n}^{(1)}+s(s-1)}-\frac{1}{\lambda_{n}^{(1)}-\frac{1}{4}+\beta^{2}}\right) \\
& +(2 h-2) \sum_{k=1}^{\infty}\left(\frac{1}{\beta+k}-\frac{1}{s-\frac{1}{2}+k}\right)+(h-1)\left[\frac{1}{\beta}-\frac{1}{s-\frac{1}{2}}\right] .
\end{aligned}
$$

In the above $\lambda_{n}^{(0)}$ are the eigenvalues of $D_{0}$ and $\lambda_{n}^{(1)}$ of $D_{1}$.

From the canonical product definition of $\Gamma_{2}(s)$ in (1.13) one verifies that

$$
\frac{d}{d s}\left(\frac{1}{2 s-1} \frac{d}{d s}\left(-\log \frac{\left(\Gamma_{2}(s)\right)^{2}(2 \pi)^{s}}{\Gamma(s)}\right)\right)=\sum_{k=0}^{\infty} \frac{1}{(k+s)^{2}}
$$

and

$$
\frac{d}{d s}\left(\frac{1}{2 s-1} \frac{d}{d s}\left(-\log \left(\Gamma_{2}(s+1 / 2)\right)^{2}(2 \pi)^{s}\right)\right)=\frac{1}{2(s-1 / 2)^{2}}+\sum_{k=1}^{\infty} \frac{1}{(k+s-1 / 2)^{2}} .
$$

We define

$$
\begin{aligned}
& Z_{\infty, 0}(s)=\left(\frac{\left(\Gamma_{2}(s)\right)^{2}(2 \pi)^{s}}{\Gamma(s)}\right)^{2 h-2}, \\
& Z_{\infty, 1}(s)=\left(\Gamma_{2}^{2}\left(s+\frac{1}{2}\right)(2 \pi)^{s}\right)^{2 h-2} .
\end{aligned}
$$

Differentiating (2.11) and using (2.12), (2.13), (2.14), we have

$$
\frac{d}{d s}\left(\frac{1}{2 s-1} \frac{d}{d s}\left(\frac{Z_{v}^{\prime}}{Z_{v}}(s)+\frac{Z_{\infty, v}^{\prime}}{Z_{\infty, v}}(s)\right)\right)=\sum_{n=0}^{\infty} \frac{2 s-1}{\left(\lambda_{n}^{(v)}+s(s-1)\right)^{2}}, \quad v=0,1 .
$$

This together with (2.7) yield

$$
\frac{d}{d s}\left(\frac{1}{2 s-1} \frac{d}{d s}\left(\frac{Z_{v}^{\prime}}{Z_{v}}(s)+\frac{Z_{\infty, v}^{\prime}}{Z_{\infty, v}}(s)\right)\right)=\frac{d}{d s}\left(\frac{1}{2 s-1} \frac{d}{d s} \log \operatorname{det}\left(D_{v}+s(s-1)\right)\right) .
$$


Hence

$$
\operatorname{det}\left(D_{v}+s(s-1)\right)=e^{E_{\nu}+F_{v}(s)(s-1)} Z_{v}(s) Z_{v, \infty}(s)
$$

for some constants $E_{v}, F_{v}, v=0,1$.

To evaluate these constants we consider the behavior of the logarithms of both sides of (2.16) as $s \rightarrow \infty$. The left-hand side has asymptotic expansion given in (2.10). The right-hand side gives

$$
E_{v}+F_{v} s(s-1)+\log Z_{v, \infty}(s)+o(1)
$$

as $s \rightarrow \infty$, since $\log Z(s) \rightarrow 0$ with $s \rightarrow \infty$ [as is apparent from the product definition (1.12)]. The behavior of $\log Z_{v, \infty}(s)$ as $s \rightarrow \infty$ is easily determined from the "Stirling series," see [B],

$$
\log \Gamma_{2}(x+1)^{-1}=\frac{x}{2} \log 2 \pi+\zeta^{\prime}(-1)-\frac{3}{4} x^{2}+\left(\frac{x^{2}}{2}-\frac{1}{12}\right) \log x+o(1) \quad \text { as } \quad x \rightarrow \infty .
$$

Using this and the previous comments we obtain for the $S(1)$ case,

$$
\begin{aligned}
-\log \operatorname{det}\left(D_{1}+s(s-1)\right)= & (2 h-2)\left[-\frac{E_{1}}{2 h-2}-\frac{1}{2} \log 2 \pi+2 \zeta^{\prime}(-1)-1 / 4\right. \\
& -\left(\frac{F_{1}}{2 h-2}+3 / 2\right) s(s-1)+\frac{s(s-1)}{2} \log s(s-1) \\
& \left.+\frac{1}{24} \log s(s-1)\right]+o(1) \text { as } \quad s \rightarrow \infty .
\end{aligned}
$$

Comparing (2.19) with (2.10) gives

$$
\begin{aligned}
\alpha & =h-1, \quad F_{1}=-(2 h-2), \quad \beta=-\frac{(h-1)}{12}, \\
E_{1} & =(2 h-2)\left(2 \zeta^{\prime}(-1)-1 / 4-1 / 2 \log 2 \pi\right) .
\end{aligned}
$$

One does the same for $v=0$, and finds

$$
\begin{aligned}
& F_{0}=-(2 h-2)=F_{1}, \\
& E_{0}=E_{1} .
\end{aligned}
$$

The evaluation of these constants together with (2.16) gives Theorem 1. We turn to the proof of the corollary. Clearly,

$$
\operatorname{det}\left(D_{0}+s(s-1)\right)=s(s-1) \prod_{k=1}^{\infty}\left(\lambda_{k}^{(0)}+s(s-1)\right)
$$

and

$$
\operatorname{det}\left(D_{1}+s(s-1)\right)=(s-1 / 2)^{2 N} \prod_{k=N}^{\infty}\left(\lambda_{k}^{(1)}+s(s-1)\right) .
$$


Hence

$$
\begin{aligned}
\operatorname{det}^{\prime}\left(D_{0}\right) & =\left.\frac{d}{d s}\left(\operatorname{det}\left(D_{0}+s(s-1)\right)\right)\right|_{s=1}, \\
\operatorname{det}^{\prime}\left(D_{1}+1 / 2(1 / 2-1)\right) & =\left.\frac{1}{(2 N) !} \frac{d^{2 N}}{d s^{2 N}}\left(\operatorname{det}\left(D_{1}+s(s-1)\right)\right)\right|_{s=1 / 2} .
\end{aligned}
$$

The right-hand sides of (2.23) may be evaluated in terms of Selberg Zeta Functions using Theorem 1. Indeed, since $Z_{0}(s)$ and $Z_{1}(s)$ have, a simple zero at $s=1$, respectively a zero of order $2 N$ at $s=1 / 2$, the corollary follows at once. [Recall $\left.\Gamma_{2}(1)=1.\right]$

Acknowledgements. The author would like to thank M. Peskin for interesting discussions and I. Vardi for introducing him to the double gamma function.

\section{References}

[B] Barnes, E.W.: The theory of the G-function. Q. J. Math. 31, 264-314 (1900)

[D-P 1] D'Hoker, E., Phong, D.H.: Multiloop amplitudes for the bosonic Polyakov string. Nucl. Phys. B 269, 205-234 (1986)

[D-P 2] D'Hoker, E., Phong, D.H.: On determinants of Laplacians on Riemann surfaces. Commun. Math. Phys. 104, 537-545 (1986)

[G] Gilkey, P.: The index theorem and the heat equation. Publish or Perish, Math. Lecture Series 4, 1974

[H] Hejhal, D.A.: The Selberg trace formula for $\operatorname{PSL}(2, \mathbb{R})$, Vol. 1. S.L.N. 548

[KIE] Kierlanczyk, M.: Determinants of Laplacians. Ph.D. thesis, M.I.T. 1986

[K 2] Kinkelin: Crelle LVII, 133, 134

[P] Polyakov, A.M.: Quantum geometry of bosonic strings. Phys. Lett. 103 B, 207 (1981)

[R-S] Ray, D., Singer, I.M.: Analytic torsion for complex manifolds. Ann. Math. 98, 154 (1973)

[S] Selberg, A.: Harmonic analysis and discontinuous groups in weakly symmetric Riemannian spaces with applications to Dirichlet series. J. Indian Math. Soc. 20, 47-87 (1956)

[VA 1] Vardi, I.: Values of Dirichlet $L$-functions and multiple gamma functions. Preprint 1985

[VA 2] Vardi, I.: A generalization of $\Gamma(1 / 2)=\sqrt{\pi}$. Preprint

[VI] Vignéras, M.F.: L'équation fonctionelle de la fonction zéta de Selberg do groupe modulare $S L(2, \mathbb{Z})$. Société arithmétique de France. Astérisque 61, 235-249 (1979)

Communicated by B. Simon

Received August 11, 1986; in revised form November 6, 1986

Note added in proof. Professors Phong and D'Hoker have informed me that they have also derived Corollary 1 by different methods. The method of deriving the relation in Corollary 1 for $\Delta_{0}$, described in this note, has been independently found by Voros in "Spectral functions, special functions, and the Selberg zeta function" preprint 1986. 